\title{
Avaliação da fertilidade de gemas de variedades de uvas americanas e híbridas cultivadas no Planalto Norte Catarinense
}

\author{
Assessing bud fruitfulness of American and hybrid grapes grown in the Northern Highlands \\ of Santa Catarina
}

\section{Douglas André Würz*, Maíra Alves Pinto, Alcemir Nabir Kowal, Thalia Aparecida Silva Maciel, Simone de Oliveira, Rabechlt Stange Almeira, Ramilton Manuel Ribeiro, Daniele Moreira Ribeiro}

Instituto Federal de Santa Catarina, Canoinhas, SC, Brasil. * Autor para correspondência: douglaswurz@hotmail.com

Submissão: 18/01/2020 / Aceite: 02/09/2020

\begin{abstract}
RESUMO
Apesar da importância da fertilidade de gemas para a produtividade e para indicação do melhor método de poda, estes dados são inexistentes na região do Planalto Norte Catarinense, e nesse contexto, o presente trabalho tem como objetivo avaliar a fertilidade de gemas de variedades de uvas americanas e híbridas cultivadas no Planalto Norte Catarinense. O presente projeto foi realizado durante as safras 2018 e 2019, em um vinhedo comercial, situado na localidade de Pedras Brancas, município de Canoinhas, Santa Catarina. Para o desenvolvimento do trabalho foram utilizadas plantas das variedades: Bordô, Niágara Branca, Casca Dura e Grano d'Oro. Coletou-se 20 ramos do ano de cada tratamento, no período de endodormência das plantas, e cada segmento do ramo contendo uma gema foi disposto em bandejas de isopor com espuma fenólica hidratada. As bandejas foram colocadas em casa de vegetação com temperatura e umidade controladas. As gemas foram classificadas em férteis ou não férteis de acordo com a presença ou ausência da inflorescência. De acordo com os dados observados no presente estudo, observa-se que as variedades Casca Dura, Niágara Branca, Bordô e Grano D'Oro apresentam boa adaptabilidade, apresentando alta fertilidade de gemas, com destaque para as gemas das porções medianas e apicais dos ramos. Dessa forma, recomenda-se a poda mista destas variedades, para obtenção de melhores produtividades nas condições do Planalto Norte Catarinense.
\end{abstract}

PALAVRAS-CHAVE: Vitis labrusca L., poda mista, Bordô, Niágara Branca, Casca Dura, Grano D’Oro.

\begin{abstract}
Although bud fruitfulness is important to yield and depends on the most appropriate pruning method, no such information is available for the Northern Highlands of Santa Catarina in Brazil. Therefore, this study proposes to evaluate the bud fruitfulness of American and hybrid grape varieties cultivated in this region. This experiment was conducted in a commercial vineyard located in Pedras Brancas, in the municipality of Canoinhas, Santa Catarina, during the 2018 and 2019 vintages. The study included plants of the following varieties: Ives Noir, White Niagara, Goethe, and Grano d'Oro. Twenty canes from the year of each treatment were collected during the endodormancy phase, and each cane segment containing one bud was arranged in Styrofoam trays with hydrated phenolic foam. The trays were placed in a greenhouse where temperature and humidity were kept under control. The buds were classified as fruitful or unfruitful based on the presence or absence of inflorescence. According to the data in this study, the Goethe, White Niagara, Ives Noir, and Grano D'Oro varieties presented good adaptability as they had high bud fruitfulness, especially the buds in the middle and apical sections of the canes. Thus, mixed pruning of these varieties is recommended to obtain better yields under the conditions of the Northern Highlands of Santa Catarina.
\end{abstract}

KEYWORDS: Vitis labrusca L., mixed pruning, Ives Noir, White Niagara, Goethe, Grano D'Oro.

A viticultura brasileira é marcada pela sua diversidade, tanto de material genético quanto de regiões produtoras (CAMARGO et al. 2011), e nessa diversidade de regiões surge o Planalto Norte Catarinense com grande potencial no cultivo de uvas americanas (Vitis labrusca L.) e híbridas para processamento. De acordo com BRIGHENTI et al. (2018), observa-se que a demanda por estas variedades não é alcançada pelos produtores do estado de Santa Catarina. Em um levantamento realizado por WÜRZ (2018a), 
verificam-se que o grande volume de vinhos brasileiros comercializados são os vinhos de mesa, aqueles produtos originários de variedades americanas e híbridas, em especial Isabel, Niágara e Bordô, variedades que também são amplamente utilizadas para elaboração de suco de uva.

As variações na produtividade são umas das maiores incertezas na produção vitivinícola, e nesse contexto, a fertilidade de gemas permanece como um fator determinante nos estudos da produtividade das culturas (SÁNCHEZ \& DOKOOZLIAN 2005). A fertilidade é uma característica genética das variedades de videira, e significa a capacidade de diferenciação de gemas vegetativas em frutíferas, o que determinará sua produtividade (LOPEZ-MIRANDA et al. 2000), sendo que cada variedade apresenta um padrão de fertilidade de gemas ao longo do ramo, o conhecimento da posição das gemas férteis é de fundamental importância na definição do tipo de poda a ser empregada no vinhedo (LEÃO \& SILVA 2003).

Apesar da importância da fertilidade de gemas para a produtividade e para indicação do melhor método de poda, esta informação é inexistente na região do Planalto Norte Catarinense, e nesse contexto, o presente trabalho teve como objetivo avaliar a fertilidade de gemas de variedades de uvas americanas e híbridas cultivadas no Planalto Norte Catarinense.

O presente projeto foi realizado durante os ciclos 2018/2019 e 2019/2020, em um vinhedo comercial, situado na localidade de Pedras Brancas, município de Canoinhas, Santa Catarina. A região é caracterizada por temperatura média anual entre 17 e $18^{\circ} \mathrm{C}$, precipitação de 1.500 a $1.700 \mathrm{~mm}$ em média, relevo plano a ondulado e solos de média fertilidade.

Para o desenvolvimento do trabalho foram utilizados plantas das variedades: Bordô, Niágara Branca, Casca Dura e Grano d'Oro, enxertados sobre VR 043-43. O vinhedo utilizado foi implantado em 2015, e caracteriza-se por apresentar plantas espaçadas 1,5 x 3,0 m, em filas dispostas no sentido Norte - Sul, conduzidas em manjedoura, podadas no sistema de poda mista, com média de 60 gemas por planta. Para a avaliação da fertilidade de gemas, foram coletados 20 ramos do ano de cada tratamento, no período de endodormência, após a maturação das gemas, durante o inverno de 2018 e 2019 . O material vegetativo dormente foi levado imediatamente ao Instituto Federal de Santa Catarina, Campus Canoinhas. As gemas foram individualizadas e separadas de acordo com suas posições no ramo em gemas basais ( $1^{\underline{a}}$ a $3^{\underline{a}}$ ), gemas medianas ( $4^{\mathrm{a}}$ a $7^{\mathrm{a}}$ ) e gemas apicais $\left(8^{\mathrm{a}}\right.$ a $\left.10^{\mathrm{a}}\right)$. Cada segmento do ramo contendo uma gema foi disposto em bandejas de isopor com espuma fenólica hidratada.

As gemas foram classificadas em férteis ou não férteis de acordo com a presença ou ausência da inflorescência. O delineamento experimental utilizado foi em blocos ao acaso, com quatro blocos e cinco ramos por blocos. As variáveis foram submetidas à análise de variância (ANOVA) e quando detectadas efeitos de tratamento, procedeu-se o teste de comparação de médias pelo Teste Tukey a 5\% de probabilidade de erro.

Para a melhor compreensão da fertilidade das quatro variedades de videira avaliadas, classificou-se a fertilidade de gemas em: a) percentual de gemas férteis (\%) (Tabela 1) e b) número de cachos por gema (Tabela 2).

Em relação ao percentual de gemas férteis, observou-se comportamento similar entre as quatro variedades avaliadas, nas diferentes posições do ramo (gemas basais, medianas e apicais). Para as variedades Bordô e Grano D'Oro observou-se fertilidade de 100\%, nas duas safras avaliadas. As variedades Casca Dura e Niágara Branca apresentaram fertilidade de 99\%, nas duas safras avaliadas, não diferindo estatisticamente das variedades Bordô e Grano D'Oro (Tabela 1). A fertilidade total pode oferecer uma informação a respeito da adaptação de uma variedade a um determinado ambiente, e de acordo com os resultados, fica evidenciado a boa adaptação destas variedades às condições do Planalto Norte Catarinense.

Em relação a fertilidade de gemas, avaliada pelo número de cachos gema ${ }^{-1}$, observou-se influência das variedades avaliadas, conforme descrito na Tabela 2. Nas duas safras avaliadas, 2017/2018 e 2018/2019, observaram-se maior fertilidade de gemas nas variedades Bordô e Grano D'Oro em relação às variedades Casca Dura e Niágara Branca, tanto para as gemas basais quanto as gemas medianas e apicais. Ressalta-se que em todas as posições das gemas nos ramos, as variedades Bordô e Grano D'Oro apresentaram mais que dois cachos ramo ${ }^{-1}$. Apesar do menor número de cachos gema ${ }^{-1}$ das variedades Niágara Branca e Casca Dura, ressalta-se que o valor observado para estas variedades é satisfatório, visto que em todas as situações observou-se valor igual ou superior a 1,5 cachos gema ${ }^{-1}$.

Para as quatro variedades avaliadas, observou-se maior número de cachos gema ${ }^{-1}$ nas gemas medianas e apicais em relação às gemas basais (Tabela 2), com destaque para as variedades Bordô e Grano D'Oro que apresentaram 2,6 cachos nas gemas apicais. A tendência de aumento da fertilidade a partir da porção menos basal das varas é consequência de um maior acúmulo de carboidratos nesta região 
(WINKLER 1965). Segundo BRIGHENTI et al. (2017), adaptando o tipo de poda com a posição das gemas mais férteis é possível aumentar a produtividade, pois haverá um aumento no número de gemas férteis ao adotar o sistema de poda adequado para cada variedade.

Tabela 1. Fertilidade de gemas basais, medianas e apicais (\%) de variedades de uvas americanas e híbridas cultivadas no Planalto Norte Catarinense, ciclos 2017/2018 e 2018/2019.

Table 1. Fruitfulness of basal, mid-cane, and apical buds (\%) of American and hybrid grape varieties cultivated in the Northern Highlands of Santa Catarina, 2017/2018 and 2018/2019 cycles.

\begin{tabular}{lcccr}
\hline Variedade & \multicolumn{4}{c}{ Fertilidade de Gemas (\%) } \\
\cline { 2 - 5 } & $\begin{array}{c}\text { Gemas Basais } \\
\left(1^{\mathrm{a}} \mathrm{a} 3^{\mathrm{a}}\right)\end{array}$ & $\begin{array}{c}\text { Gemas Medianas } \\
\left(4^{\mathrm{a}} \mathrm{a} 6^{\mathrm{a}}\right)\end{array}$ & $\begin{array}{c}\text { Gemas Apicais } \\
\left(7^{\mathrm{a}} \mathrm{a} 10^{\mathrm{a}}\right)\end{array}$ & Média \\
\hline Niágara Branca & $98 \mathrm{~ns}$ & $2017 / 2018$ & 99 \\
Bordô & $100 \mathrm{~ns}$ & 100 & 99 & 100 \\
Grano D'Oro & $100 \mathrm{~ns}$ & 100 & 100 & 100 \\
Casca Dura & $97 \mathrm{~ns}$ & 100 & 100 & 99 \\
CV (\%) & 2,3 & 0,0 & 100 & 1,1 \\
Niágara Branca & $99 \mathrm{~ns}$ & $2018 / 2019$ & 1,2 & 99 \\
Bordô & $100 \mathrm{~ns}$ & 99 & 100 & 100 \\
Grano D'Oro & $100 \mathrm{~ns}$ & 100 & 100 & 100 \\
Casca Dura & $98 \mathrm{~ns}$ & 100 & 100 & 99 \\
CV (\%) & 2,2 & 0,5 & 98 & 1,0 \\
\hline
\end{tabular}

ns = não significativo pela análise de variância (ANOVA) a 5\% de probabilidade de erro.

Tabela 2. Fertilidade de gemas basais, medianas e apicais (número de cachos gemas ${ }^{-1}$ ) de variedades de uvas americanas e híbridas cultivadas no Planalto Norte Catarinense, ciclos 2017/2018 e 2018/2019.

Table 2. Fruitfulness of basal, mid-cane, and apical buds (number of bud clusters ${ }^{-1}$ ) of American and hybrid grape varieties cultivated in the Northern Highlands of Santa Catarina, 2017/2018 and 2018/2019 cycles.

\begin{tabular}{lcccc}
\hline \multirow{2}{*}{ Variedade } & \multicolumn{3}{c}{ Fertilidade de Gemas (número de cachos gemas $\left.{ }^{-1}\right)$} \\
\cline { 2 - 5 } & $\begin{array}{c}\text { Gemas Basais } \\
\left(1^{\mathrm{a}} \mathrm{a} \mathrm{3}^{\mathrm{a}}\right)\end{array}$ & $\begin{array}{c}\text { Gemas Medianas } \\
\left.\left(4^{\mathrm{a}} \mathrm{a} \mathrm{6}\right)^{\mathrm{a}}\right)\end{array}$ & $\begin{array}{c}\text { Gemas Apicais } \\
\left(7^{\mathrm{a}} \mathrm{a} 10^{\mathrm{a}}\right)\end{array}$ & Média \\
\hline Niágara Branca & $1,6 \mathrm{Bb}^{*}$ & $2017 / 2018$ & $1,8 \mathrm{Ab}$ \\
Bordô & $1,6 \mathrm{Bb}$ & $1,8 \mathrm{Ab}$ & $1,9 \mathrm{Ab}$ & $1,9 \mathrm{Ab}$ \\
Grano D'Oro & $2,1 \mathrm{Ba}$ & $1,9 \mathrm{Ab}$ & $2,0 \mathrm{Ab}$ & $2,3 \mathrm{Aa}$ \\
Casca Dura & $2,1 \mathrm{Ba}$ & $2,4 \mathrm{Aa}$ & $2,6 \mathrm{Aa}$ & $2,3 \mathrm{Aa}$ \\
CV (\%) & 7,5 & $2,4 \mathrm{Aa}$ & $2,6 \mathrm{Aa}$ & 7,3 \\
& & 7,7 & 9,2 & $1,7 \mathrm{ABb}$ \\
Niágara Branca & $1,5 \mathrm{Bb}$ & $2018 / 2019$ & $1,9 \mathrm{Ab}$ & $1,9 \mathrm{Ab}$ \\
Bordô & $1,6 \mathrm{Bb}$ & $1,8 \mathrm{Ab}$ & $2,0 \mathrm{Ab}$ & $2,4 \mathrm{Aa}$ \\
Grano D'Oro & $2,0 \mathrm{Ba}$ & $1,9 \mathrm{Ab}$ & $2,6 \mathrm{Aa}$ & $2,5 \mathrm{Aa}$ \\
Casca Dura & $2,1 \mathrm{Ba}$ & $2,5 \mathrm{Aa}$ & $2,6 \mathrm{Aa}$ & 7,1 \\
CV (\%) & 7,1 & $2,5 \mathrm{Aa}$ & 8,5 & 6,9 \\
\hline
\end{tabular}

*Médias seguidas da mesma letra maiúscula, na linha, e da mesma letra minúscula, na coluna, não diferem entre si pelo teste Tukey a $5 \%$ de probabilidade de erro. ns = não significativo pela análise de variância $($ ANOVA) a $5 \%$ de probabilidade de erro.

Trabalhos realizados por MUNHOZ et al. (2016), com as variedades italianas Greco di Tufo e Coda di Volpe; por ROSA et al. (2014) com as variedades Cabernet Sauvignon e Nebbiolo; por WÜRZ et al. (2018b) e WÜRZ et al. (2019) com as variedades Cabernet Sauvignon, Nebbiolo, Chardonnay, Sauvignon Blanc, Merlot e Cabernet Franc e por MENEGUZZI et al. (2020), com 26 variedades indicam uma maior fertilidade 
de gemas na porção mediana e apical do ramo, e com isso, indica-se realizar um poda longa ou mista nestas variedades, propiciando maior produtividade do vinhedo.

De acordo com os dados observados no presente estudo, observa-se que as variedades Casca Dura, Niágara Branca, Bordô e Grano D'Oro apresentam boa adaptabilidade, apresentando alta fertilidade de gemas, com destaque para as gemas das porções medianas e apicais dos ramos. Dessa forma, recomenda-se a poda mista destas variedades, para obtenção de melhores produtividades nas condições do Planalto Norte Catarinense.

\section{REFERÊNCIAS}

BRIGHENTI AF et al. 2017. Ecophysiology of three Italian cultivars subjected to two pruning methods in Santa Catarina, Brazil. Acta Horticulturae 1157: 381-388.

BRIGHENTI AF et al. 2018. Qualification of 'Bordô' grape clones in Vale do Rio do Peixe, in the state of Santa Catarina, Brazil. Pesquisa Agropecuária Brasileira 53: 800-808.

CAMARGO UA et al. 2011. Progressos da viticultura brasileira. Revista Brasileira de Fruticultura 33: 144-149.

LEÃO PCS \& SILVA EEG. 2003. Brotação e fertilidade de gemas em uvas sem sementes no Vale do São Francisco. Revista Brasileira de Fruticultura 25: 375-378.

LOPEZ-MIRANDA SL et al. 2000. Fluctuations of yield components in Verdejo vineyards as affected by the bud position, either in spurs or on canes. In: 6th International Symposium on Grapevine Physiology and Biotechnology. Anais... Heraklion: Book of Abstracts.

MENEGUZZI A et al. 2020. Fertility of buds and pruning recommendation of different grapevine varieties grown in altitude regions of Santa Catarina State, Brazil. Revista Ceres 67: 30-34.

MUNHOZ B et al. 2016. Fertilidade e Análise de gemas das videiras Greco di Tufo, Coda di Volpe e Viognier cultivadas em São Joaquim - Santa Catarina. Revista Agropecuária Catarinense 29: 68-72.

ROSA AM et al. 2014. Fertilidade e reserva de carbono e nitrogênio em gemas de ramos das viníferas 'Cabernet Sauvignon' e 'Nebbiolo'. Revista Brasileira de Fruticultura 36: 576-585.

SÁNCHEZ LA \& DOKOOZLIAN NK. 2005. Bud microclimate and fruitfulness in Vitis vinifera L. American Journal Enology and Viticiculture 56: 319-329.

WINKLER AJ. 1965. Viticultura. México: Continental. 792p.

WÜRZ DA. 2018a. Análise da comercialização de vinhos finos e de mesa no Brasil. Journal of Agronomic Science 7: 4349.

WÜRZ DA et al. 2018b. Reguladores de crescimento na fertilidade de gemas e maturação da cultivar 'Nebbiolo' em região de elevada altitude de Santa Catarina. Scientia Agraria Paranaensis 17: 90-95.

WÜRZ DA et al. 2019. Avaliação da fertilidade de gemas de uvas viníferas cultivadas em região de elevada altitude de Santa Catarina. Revista de Ciências Agroveterinárias 18: 81-86. 